\title{
Une autre visite de la BNU. La psychologie de l'espace pour guide
}

\section{Valérie Lebois}

\section{(2) OpenEdition}

\section{Journals}

Édition électronique

URL : http://journals.openedition.org/rbnu/295

DOI : $10.4000 /$ rbnu.295

ISSN : 2679-6104

\section{Éditeur}

Bibliothèque nationale et universitaire de Strasbourg

\section{Édition imprimée}

Date de publication : 23 mai 2018

Pagination : 50-60

ISSN : 2109-2761

\section{Référence électronique}

Valérie Lebois, "Une autre visite de la BNU. La psychologie de l'espace pour guide ", La Revue de la BNU [En ligne], 17 | 2018, mis en ligne le 05 septembre 2018, consulté le 12 décembre 2020. URL : http://journals.openedition.org/rbnu/295; DOI : https://doi.org/10.4000/rbnu.295

\section{(c) (1) ()}

La Revue de la BNU est mise à disposition selon les termes de la Licence Creative Commons Attribution - Pas d'Utilisation Commerciale - Partage dans les Mêmes Conditions 4.0 International. 


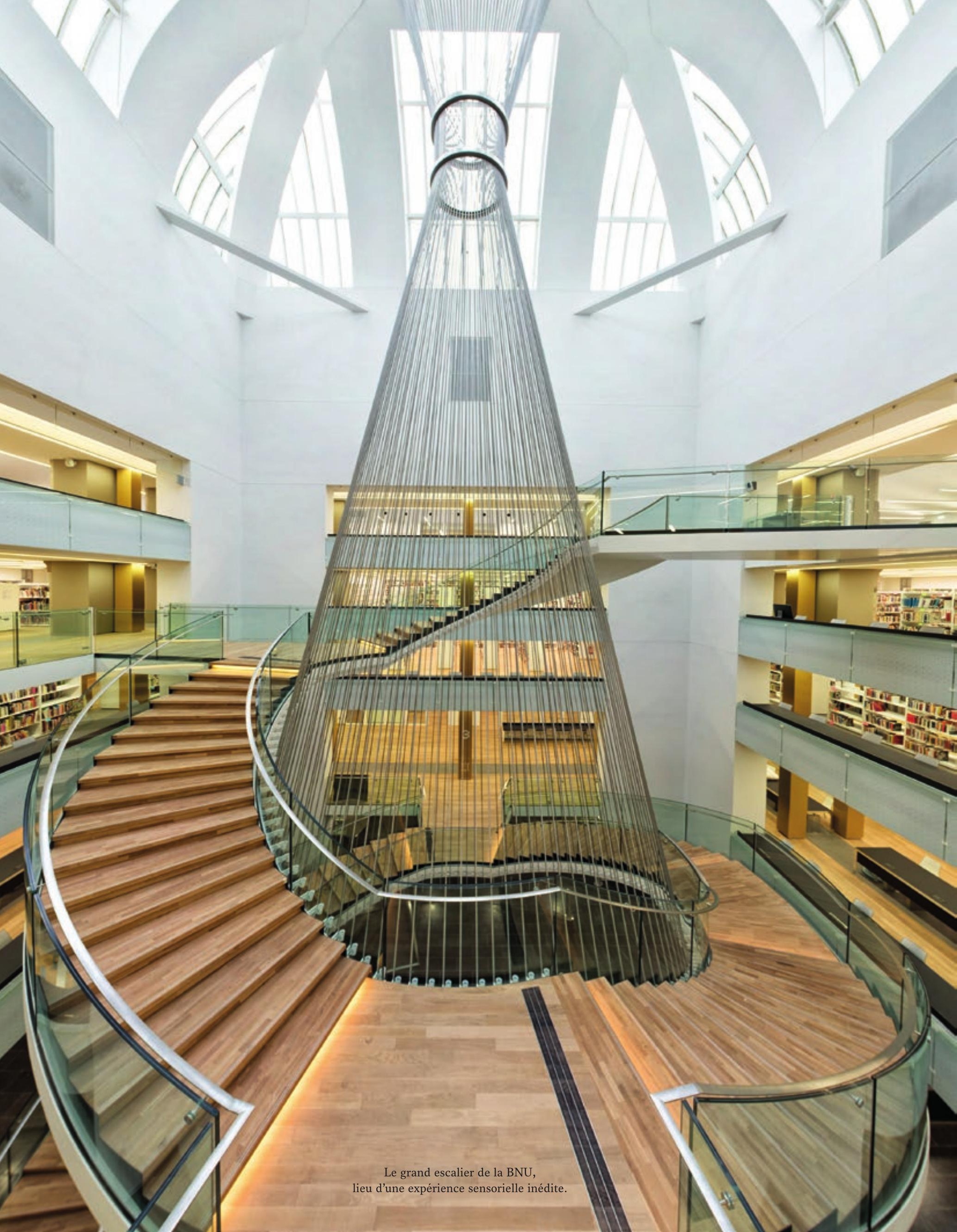




\title{
UNE AUTRE VISITE DE LA BNU. LA PSYCHOLOGIE DE L'ESPACE POUR GUIDE
}

\author{
PAR VALÉRIE LEBOIS
}

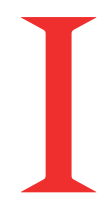

nviter un groupe de visiteurs à pousser un grand cri depuis le sculptural escalier de la Bibliothèque nationale et universitaire de Strasbourg nous aide-t-il à mieux comprendre les liens que nous entretenons avec cet édifice ? Cette action atypique renvoie à un thème cher à la psychologie de l'espace : étudier les modes d'exploration de l'individu pour expérimenter un lieu afin de le faire sien à des degrés divers.

Nous sommes toutefois bien conscients qu'en conviant les visiteurs à ce surgissement sonore, nous sommes en décalage avec les usages et les modes d'appropriation habituellement observés à la bibliothèque. Cette démarche " décalée " a deux objectifs : d'abord ré-interroger, au-delà de l'organisation sociale et conventionnelle des lieux, les potentialités bâties en termes d'actions individuelles et collectives; d'autre part, attirer l'attention sur notre rapport plurisensoriel à l'espace et ainsi rétablir la portée des autres sens (sonore, olfactif, tactile, kinesthésique...), en dehors du très valorisé registre visuel. Il nous intéresse en effet de comprendre ce que l'architecture fait au corps de l'usager et vice-versa.

Nous proposons dans cet article de revenir sur les principes qui ont motivé la conception de cette visite atypique. Précisons qu'elle a été élaborée et réalisée avec la chorégraphe et danseuse Vivian Fritz-Roa et a bénéficié de la participation de Patrick Romieu, anthropologue du sonore. Nous détaillerons leur rôle respectif dans les différentes propositions qui ont jalonné la visite. Enfin, nous livrerons les réflexions auxquelles nous a conduit cet exercice. Nous enrichirons également notre propos grâce aux enseignements et aux recherches ${ }^{1}$ que nous développons depuis plusieurs années sur l'expérience sensible de l'architecture.

\section{INSPIRATIONS}

L'idée de cette visite est née de la demande des organisateurs du colloque consacré à Abraham Moles en septembre dernier à l'Université de Strasbourg. L'objectif était de proposer une lecture de la bibliothèque sous l'angle des théories développées par ce penseur transdisciplinaire du $20^{e}$ siècle qui, formé initialement à l'ingénierie acoustique, s'est imposé dans le champ des sciences sociales en couvrant une multiplicité de sujets.

Cette demande nous a séduit, d'abord parce qu'elle nous permettait de prolonger la réflexion du séminaire de recherche que nous avions organisé en mai dernier sur une actualisation des liens entre les travaux d'A. Moles et l'architecture ${ }^{2}$, et aussi parce qu'elle faisait écho à la démarche que nous menons dans le cadre d'un enseignement de master sur le rapport corps/espace au sein des bibliothèques. 


\section{Sur les pas d'Abraham Moles}

Pour revenir à Abraham Moles, rappelons que son histoire est étroitement liée à l'introduction des sciences sociales au sein de l'École d'architecture de Strasbourg à la fin des années $1960^{3}$. Bien qu'il n'ait pas directement enseigné dans cette école, il a, depuis son poste de professeur de sociologie, puis de psychologie sociale à l'Université de Strasbourg, préparé le terrain. D'abord en développant des thématiques de recherche en prise directe avec les préoccupations architecturales et urbaines naissantes. Ensuite, en constituant

et en formant un pôle de collaborateurs

devenus depuis enseignants et chercheurs au sein même des écoles, notamment à Strasbourg.

C'est particulièrement son enseignement sur la psychologie de l'espace qui a attiré, de son vivant, les architectes et retient aujourd'hui notre attention. Cet enseignement, consigné plus tard dans plusieurs ouvrages ${ }^{4}$, privilégie le point de vue de celui qui perçoit, vit son environnement, son habitat, sa ville. Il montre comment ce système de lecture « égocentré » de l'espace entre en tension avec une approche euclidienne ou cartésienne de ce dernier. En ce sens, Moles s'adresse directement aux professionnels de l'aménagement en les interpellant sur leur propre système de représentations. Il expose un problème fondamental auquel toute volonté d'apport psychosociologique dans le domaine architectural ne cesse de se heurter : comment, en partant d'une « analyse intuitive et volontiers poétique de l'idée d'espace ", faisant toute sa place à l'irrationalité de l'être, peut-on tirer une quelconque « règle de raison à l'usage du constructeur d'espace ${ }^{5}$ ?

C'est en abordant frontalement cette question et en recherchant des modes d'articulation entre l'espace métrique de l'aménageur et l'espace sensible de l'habitant, que les théories d'A. Moles offrent des points de passage entre les sciences sociales et l'architecture qui restent, selon nous, toujours fructueux à considérer.
Parmi les clefs de lecture que nous avons retenues dans notre proposition de visite de la BNU, il y a celle qui concerne la mise en exergue du corps de l'individu et de son rôle primordial dans la découverte et l'expérience de l'espace. En effet, Moles ne s'arrête pas aux phénomènes perceptifs statiques qui donnent en général le beau rôle au sens de la vision, il intègre une dimension active qui le conduit à insister sur le mouvement : «L'homme expérimente avec son corps, avec ses gestes, et non avec ses yeux $[. ..]{ }^{6}{ }^{6}$; "Le mouvement est l'un des facteurs principaux de l'appropriation de l'espace par l'être, l'espace n'existe phénoménologiquement que par les mouvements qui le remplissent $"{ }^{7}$. Il regrette ainsi que « l'architecture se soit imposée au titre d'un art visuel des volumes, au lieu d'un art à pratiquer ${ }^{8}$, car pour Moles il s'agit bien de considérer l'architecture comme une « forme à pratiquer ${ }^{9}$ plutôt qu'une œuvre à voir au sens strict. Ce qui, corrélativement, lui permet d'adopter le point de vue du concepteur et de chercher la manière dont il peut agir sur la valeur et l'identité d'un lieu en jouant de la variation des propriétés sensorielles de l'espace.

Moles prend l'exemple de la paroi, élément réputé « dur» de l'architecture, et l'envisage à travers ses possibilités de "discontinuité perceptive»: " construire un mur, c'est créer une variation brusque de plusieurs propriétés perceptives de l'espace, et le mur aura d'autant plus d'importance que cette variation sera en elle-même mieux ressentie psychologiquement $"{ }^{10}$. En déclinant les possibilités de discontinuité suivant plusieurs grandeurs perceptives (diamètre apparent, audition, résistance au déplacement, température, odeur ou couleur), Moles introduit une notion particulièrement opérante aux yeux des concepteurs : la notion de "gradient sensoriel », en montrant ses effets de structuration entre un dehors et un dedans, un ici et un ailleurs (valeur symbolique), entre le Moi et les Autres (valeurs sociale et culturelle). 
Définie par A. Moles en pionnier, cette appréhension "sensualisante " ${ }^{11}$ de l'expérience de l'espace fait écho à l'orientation prise depuis une vingtaine d'années au sein des formations d'architecte. Celle-ci s'appuie sur une remise en question d'une approche oculo-centrée de la lecture des lieux. Grâce au développement du champ de recherche sur les ambiances dans les écoles, l'accent est davantage mis sur l'aspect immersif de l'espace, autrement dit l'espace comme milieu de vie et " matière première de l'existence ${ }^{12}$. C'est dans cette perspective que nous avons créé un séminaire de master à l'École d'architecture de Strasbourg, en ayant pour but de sensibiliser les étudiants à la manière dont s'éprouve un lieu, à son pouvoir d'affection et à sa puissance d'imprégnation.

\section{— Explorations sensibles de la Médiathèque Malraux à Strasbourg}

Pour préparer notre visite de la BNU, nous nous sommes bien évidemment inspiré du travail que nous développons au sein de ce séminaire et de la manière dont nous le faisons évoluer au fil des années.

À la croisée de l'architecture, des sciences sociales et de la pratique artistique, notre démarche pédagogique vise à aiguiser la conscience corporelle des étudiants et à chercher avec eux les moyens de fixer ces expériences pour qu'elles puissent nourrir de manière plus explicite leur travail d'architecture. À l'heure de la généralisation de la conception assistée par ordinateur, il apparaît nécessaire de ne pas perdre le contact avec le réel, le vécu des lieux et d'apprendre à le projeter dans toute sa richesse sensorielle.

Outre la démarche, ce qui fait le lien entre ce travail et la visite de la $\mathrm{BNU}$ réside dans le fait que nous avons choisi, depuis plusieurs années, comme lieu d'« entraînement " la Médiathèque Malraux de Strasbourg. La médiathèque est en effet un programme contemporain qui parle au corps de l'usager : " elle lui propose un lieu de séjour plus ou moins prolongé, un univers protecteur, accueillant selon ses envies et son état d'esprit et donne aussi une place à ses accessoires ${ }^{13}$. De plus, la Médiathèque Malraux offre par son déploiement sur plusieurs niveaux et la diversité des publics visés une grande amplitude de mouvement ainsi que des ambiances contrastantes.
Afin de favoriser les capacités d'immersion plurisensorielles des étudiants, nous organisons deux types d'exploration de la médiathèque. La première est assurée par Vivian Fritz-Roa, danseuse-chorégraphe. Elle vise à faire prendre conscience du rôle du mouvement et de la gestuelle dans la manière dont nous vivons et nous approprions ce lieu. La seconde est proposée par Patrick Romieu, anthropologue du sonore. Elle consiste à proposer une déambulation centrée sur l'écoute afin de révéler les logiques de spatialité, l'organisation humaine et la dynamique des lieux.

Ces explorations nous ont permis de caractériser les qualités d'ambiance propres à la Médiathèque Malraux. Au-delà de ce contexte particulier, elles nous ont permis de prendre plus largement la mesure des évolutions qui traversent aujourd'hui, non sans tensions, ce type d'équipement. La bibliothèque tend en effet à devenir un " lieu de vie " ouvert sur la ville, où le rapport au livre devient secondaire et où il est davantage question de privilégier le confort d'usagers aux profils et aux envies variés ${ }^{14}$. Bien que le statut de bibliothèque " universitaire " de la BNU en fasse une catégorie à part des équipements plus généralistes, il nous paraît intéressant de poser, dans son cas aussi, ces questions d'évolution - et ce d'autant que les orientations adoptées au moment de la dernière rénovation de la BNU ne semblent pas étrangères à la prise en compte de ces nouvelles attentes.

\section{LES MOMENTS FORTS DE LA VISITE}

Prendre l'angle de la psychologie de l'espace pour organiser une visite de la BNU nous a conduit à nous démarquer d'une visite dite traditionnelle. Car dans ce type de visite, c'est en général un parti historique et esthétique qui est valorisé. Le visiteur est volontairement mis dans une position d'observateur extérieur. Le parcours proposé s'apparente à une succession de tableaux dont le choix et la délimitation prennent tout leur sens grâce aux commentaires du conférencier. La mise en valeur patrimoniale ainsi que la focalisation sur des éléments reconnus pour leur valeur artistique (rarement fonctionnelle) placent le visiteur dans un espacetemps très souvent déconnecté de l'instant présent du bâtiment et de ses usages. Le visiteur est plutôt invité à s'abstraire du contexte actuel afin de privilégier, grâce 


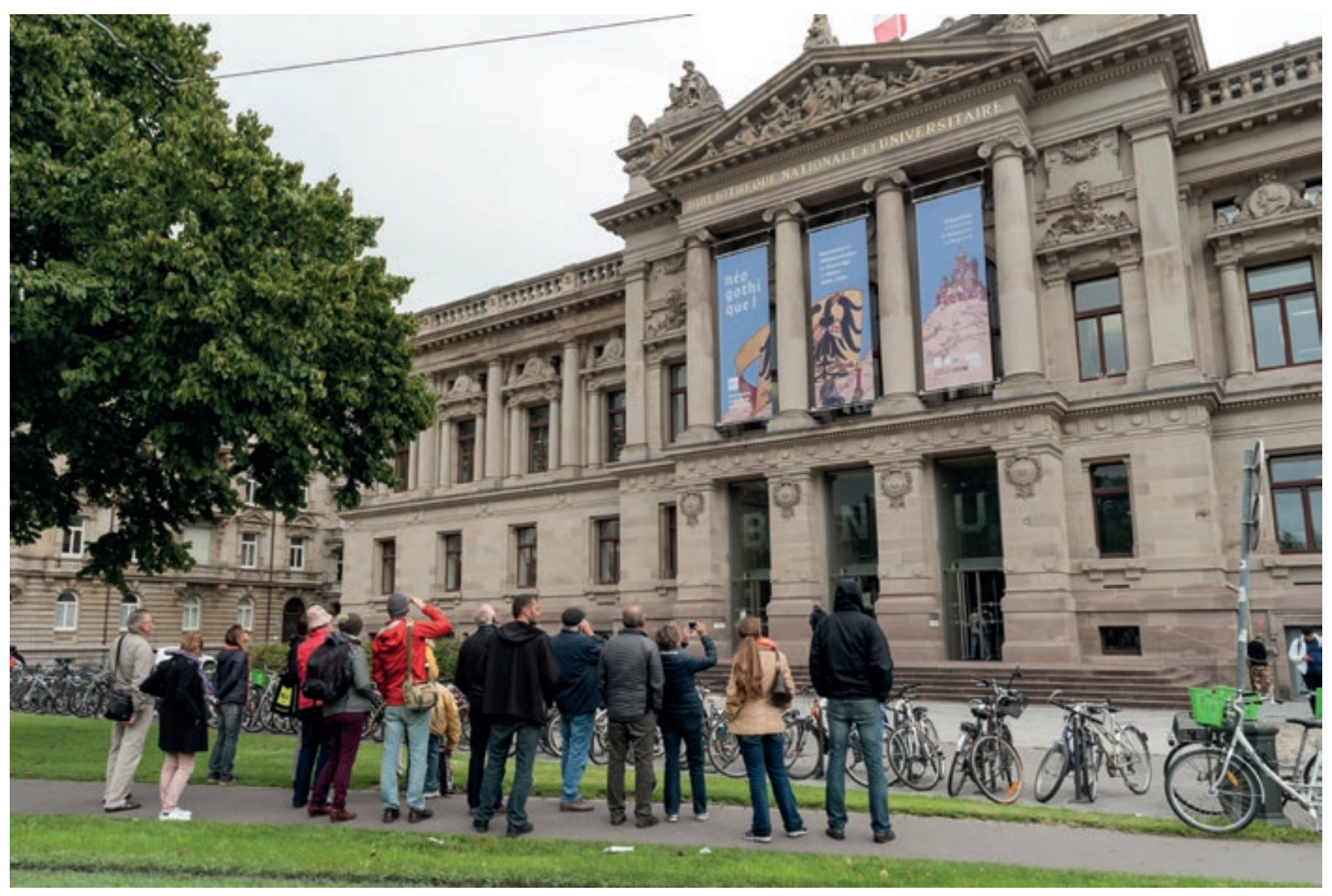

Sur le parvis de la bibliothèque 
à la stimulation de son imagination et d'un travail de conceptualisation, une attitude essentiellement contemplative du lieu. Aussi le conférencier s'adresse-t-il avant tout à l'intellect $\mathrm{du}$ visiteur et fait-il de ce dernier un spectateur à qui il est demandé bien souvent de ne " toucher qu'avec les yeux » et de se faire le plus discret possible (surtout quand le bâtiment est en fonction). Ce préalable qui préside à de nombreuses visites révèle bien cet effacement du corps au profit de l'esprit.

Nous avons choisi de renverser ce principe pour mettre au centre de notre visite l'expérience corporelle du visiteur. L'objectif est de donner à vivre l'architecture de ce bâtiment, et moins de la donner à voir. Si l'on reprenait le vocabulaire de Moles, on pourrait parler d'une approche égocentrée de l'espace, et moins allocentrée. On cherche ainsi à activer chez le visiteur son implication en tant qu'habitant, acteur et usager potentiel $\mathrm{du}$ lieu. La visite prend alors un tour différent de la visite touristique pour laquelle il est important de marquer les ruptures quotidien/hors quotidien, ordinaire/extraordinaire, laissant volontairement de côté les imbrications temporelles et catégorielles des individus ${ }^{15}$.

La tâche des différents guides a consisté à aider les visiteurs à être le plus possible à l'écoute de leurs propres sensations et à leur faire vivre en toute conscience les activités ordinaires du lieu. La visite comportait quatre propositions. Chacune avait pour but de confronter les visiteurs à des moments identifiés comme clefs dans le parcours et les habitudes des usagers.

\section{L'étape de la transformation}

La première étape de la visite commence à l'extérieur de la BNU, sur le parvis. Les participants sont invités à prendre du recul par rapport au bâtiment. Non pas pour en admirer les façades, mais pour s'imprégner du milieu urbain, en expliciter la nature. Cette phase préalable permettra, par contraste, de mieux saisir ce que d'entrer dans le bâtiment produit comme changements.

Nous proposons pour cela quelques petits exercices qui visent, les yeux fermés, à porter attention à sa posture corporelle (les appuis sur le sol, la tonicité générale), à écouter son environnement (dans son amplitude sonore, sa rythmicité), à le respirer (dans ses mouvements d'air, ses odeurs) et à le sentir dans sa densité humaine (à travers les rapports de proximité avec les autres). Cette reconnexion à soi-même et à son milieu est nécessaire pour inciter les participants à vivre avec attention ce qui va se jouer à travers le passage de ce seuil.

Entrer dans un bâtiment, quel qu'il soit, entraîne en effet des transformations dans les sensations, les comportements, aussi imperceptibles soient-ils. Au seuil de la conscience vont s'opérer de nombreux ajustements intégrant les paramètres physiques, sensoriels et sociaux du nouvel environnement. C'est inévitablement le champ perceptif qui se modifie : les cadrages visuels sont plus découpés, la température ambiante se stabilise, l'environnement sonore est moins diffus, plus contrôlé, le rapport à l'extérieur est filtré. C'est aussi la posture corporelle qui s'adapte : la tension propre à la vie publique se relâche, le rythme de marche n'est plus celui adopté en ville. C'est également le rapport aux autres qui n'est plus le même : d'autres règles tacites l'organisent.

Cette étape de l'entrée n'est-elle pas intensifiée par le statut même de la BNU ? Ce bâtiment porte en lui une mission publique forte liée à la culture et à la connaissance d'une société. Il vise à proprement parler la transformation d'individus lambda en « citoyens éclairés ». La logique de conception du bâtiment n'est pas ignorante de cette symbolique. Le dispositif architectural est nourri dans sa construction des ambitions attribuées à ce " temple " du savoir, telles que la sacralisation du livre, la célébration du bien commun ou encore l'invitation au voyage.

Les escaliers ne sont-ils pas ici les pièces maîtresses de cette transformation? Commençons par les volées de marches qui séparent les portes d'accès principales du plateau d'accueil de la bibliothèque. Comme toute marche d'escalier, celle-ci réalise une contrainte psychomotrice qui oblige l'individu à adapter sa démarche à la pente de l'escalier, aux hauteur et largeur des marches. Selon le réglage de ces paramètres, gravir les escaliers peut s'accompagner d'une certaine solennité qui, comme le montre Moles, n'est pas sans inscrire une part de sacré dans l'espace ${ }^{16}$. L'effort requis pour son ascension entraîne un surcroît d'attention pour soi et pour le lieu. 
Le phénomène attentionnel est ici amplifié par le gravissement de l'escalier principal qui prolonge ce premier parcours ascensionnel. La position centrale et ouverte du dispositif monumental confère à celui qui l'arpente une grande visibilité sur le cœur de la bibliothèque. Il peut ainsi exercer un droit de regard sur les activités qui s'y déroulent. Il crée aussi l'événement pour tous ceux qui sont installés autour. Dans ce rapport de l'observateur observé, que se joue-t-il ? Une mise en condition ? Le sentiment aigu d'une conscience de soi ?

Richard Escoffier, philosophe contemporain de l'architecture, établit un parallèle ${ }^{17}$ entre ces dispositifs centrés, caractéristiques des bibliothèques, et le panopticon ${ }^{18}$ inventé à la fin du $18^{\mathrm{e}}$ siècle par Jeremy Bentham pour surveiller les prisonniers. Il voit un rapprochement possible entre le souhait initial du philosophe anglais et le rôle attribué aux bibliothèques. En voulant donner aux détenus le sentiment d'être surveillés constamment, Bentham envisageait la prison comme une sorte de prothèse, non pas pour punir les prisonniers, mais pour les amener à se construire un surmoi, à développer une conscience morale et sociale. La bibliothèque porterait aussi en elle, à travers ces dispositifs panoptiques, le désir de la transformation d'un « être voyant " en un " être lisant » ${ }^{19}$.

En s'attachant à faire vivre pleinement aux visiteurs le parcours d'entrée dans la bibliothèque, notre but est bien de leur faire saisir, à partir de leurs sensations, l'emprise de ces dispositifs, leur « pouvoir organisateur » ainsi que leur rôle de mise en cohérence de l'expérience.

\section{Une expérience vivante des formes}

La deuxième étape de la visite consiste justement à approfondir les effets que procure la découverte de l'escalier central, devenu, depuis la rénovation, pièce majeure de la BNU. S'il peut être admiré comme un objet d'art, cet escalier donne aussi à vivre des formes, des matières qui entrent en résonance avec notre propre corporéité, autrement dit avec le poids de notre corps, sa structure squelettique, son rapport au mouvement, son sens de l'équilibre. Autant d'outils de captation qui dépassent les cinq sens traditionnels et font surgir dans l'appréhension des formes des impressions
" premières » puissantes de massivité ou de légèreté, de plasticité ou encore de dynamisme ${ }^{20}$.

Guidés par la chorégraphe de notre équipe, les participants sont donc invités à aller au-delà d'un ébahissement statique pour s'engager de manière dynamique dans l'expérience vivante des formes. Il leur est d'abord proposé d'expérimenter différents points de vue de l'escalier en se donnant une amplitude de déplacement (tout autour de l'escalier) et en variant leur posture (assise, accroupie, grandie). C'est ensuite par le développement de la gestuelle, notamment des bras et des mains, qu'ils sont encouragés à sentir les formes " retomber " dans leur corps sans négliger les effets sensuels que procure la lumière zénithale qui les baigne. La chorégraphe invite à observer la manière dont ces actions corporelles stimulent l'imaginaire. Le mouvement n'aide-t-il pas à ressentir les émotions ?

\section{Un grand cri pour révéler le silence}

La troisième étape nous ramène à l'introduction de notre article. Conduite par Patrick Romieu, anthropologue spécialiste de l'environnement sonore, elle vise à amplifier la projection de soi, de son corps dans l'espace par l'usage du cri. Pour reprendre le concept de Moles, on pourrait parler d'une recherche d'expansion des « coquilles " de l'individu dans cette mise en synergie du corps, du souffle et du son.

Positionnés par petits groupes sur les paliers les plus hauts de l'escalier central (niveaux 4, 5, 6), les participants sont invités à clamer à l'unisson un "Ah!" puissant et bref, d'abord les trois groupes séparément, puis tous ensemble.

De manière évidente, la puissance sonore envoyée réveille les propriétés acoustiques du bâti qui se révèlent ici très absorbantes et peu réverbérantes. Cela nous informe sur l'effort fourni en pratique par les usagers pour se conformer à la demande de silence requise. D'une manière générale, celle-ci questionne les rapports de lutte qu'entretiennent les organisations humaines avec les propriétés même des lieux dans lesquels elles prennent place.

D'autre part, ce «grand cri » donne à vivre aux participants le déploiement d'une énergie collective. Elle est vécue comme une libération des corps les 


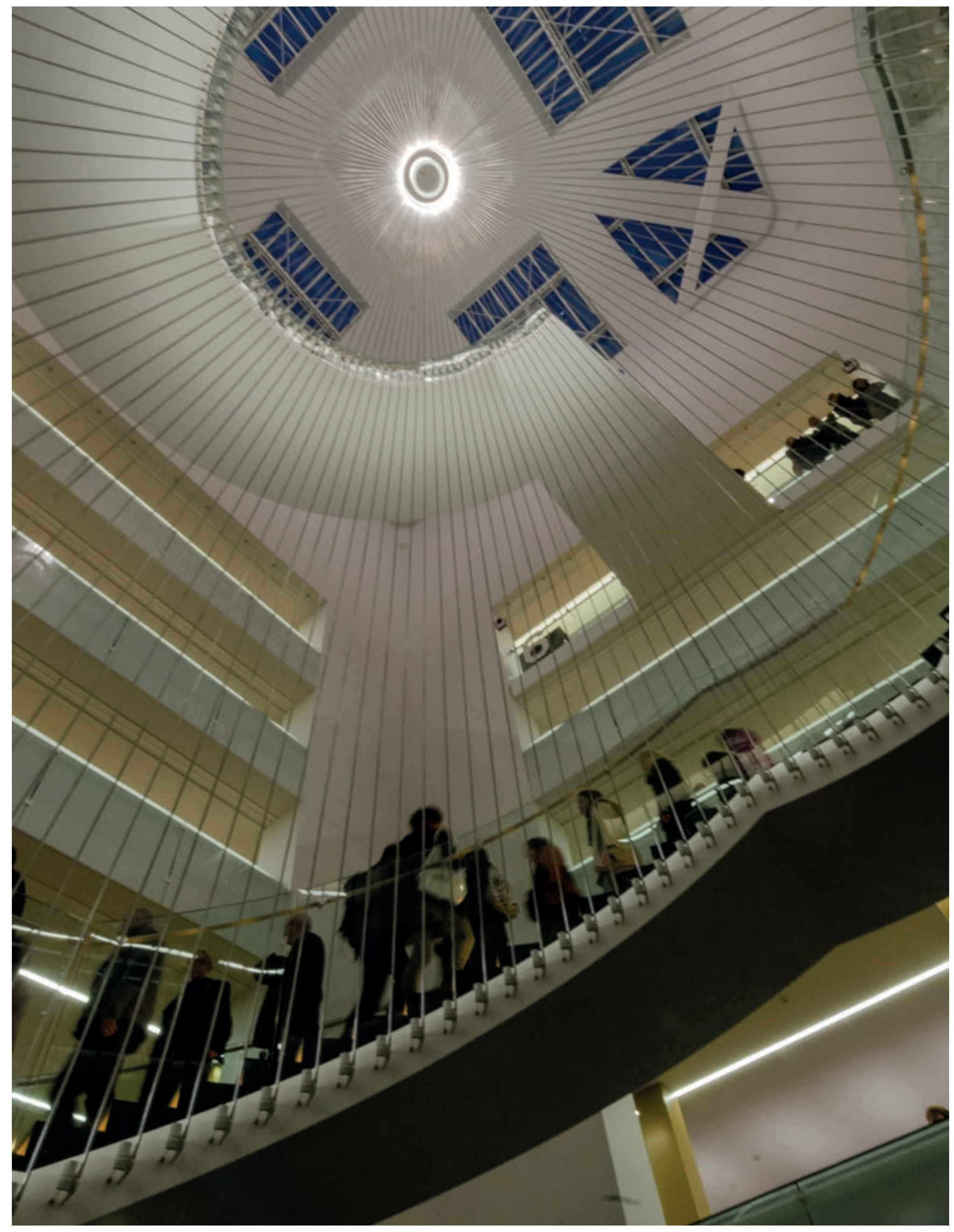

Un groupe dans l'escalier 
uns vis-à-vis des autres. C'est comme si l'exaltation provoquée par le cri permettait un délestage des conventions sociales et faisait découvrir à chacun les possibilités du groupe. En cela, elle invite à un autre mode de conquête de l'espace où prévaut la capacité à faire groupe - ce qui, par extension, nous amène à nous questionner sur les formes possibles d'expression du collectif dans un établissement où la primauté est donnée à l'individualité.

Enfin, cette irruption sonore peut aussi être interprétée comme un acte de désacralisation tant il est impensable de l'envisager dans le fonctionnement « normal » de la bibliothèque. Déchirer le silence dans une bibliothèque, c'est aller à l'encontre d'une force « ambiantale » qui s'impose aux usagers. Le "grand cri » questionne la nature de cette force qui tient beaucoup à la maîtrise de l'environnement sonore, révélant ainsi le caractère disciplinaire du son. Soulignons d'ailleurs que cette expérience a perdu de son caractère transgressif dans la mesure où elle s'est déroulée en dehors des horaires d'ouverture au public.

\section{Esprit labyrinthe, es-tu là ?}

La dernière étape consistait à profiter des groupes ainsi constitués pour les lancer dans une exploration plus autonome de la bibliothèque. Le propre de la psychologie de l'espace n'est-il pas avant tout de reconnaître à l'individu son rôle d'acteur dans la fabrique de l'espace ? Parmi plusieurs missions qu'ils avaient à accomplir, nous évoquerons celle qui visait à interroger, au sein des espaces de lecture de la bibliothèque, le concept de "labyrinthe » de Moles. Ce dernier s'est en effet intéressé à l'archétype du labyrinthe pour ce qu'il représente comme « solution topologique à l'une des dialectiques fondamentales de la psychologie de l'usage de l'espace : la dialectique entre communauté et privatisation ${ }^{21}$. Le labyrinthe rend compte pour lui d'une organisation sociale dans l'espace qui permet la densité des individus sans pour autant créer des rencontres obligatoires. Cela revient à nous demander, pour ce qui est de la bibliothèque : sur quels effets d'architecture l'usager peut-il s'appuyer pour s'isoler dans la multitude ? Cette disposition nous semblait d'autant plus intéressante à questionner que, dans sa nouvelle conception, la BNU se caractérise par des espaces très communicants avec une recherche d'ouverture et de visibilité accrue.

Chaque groupe devait proposer une mise en situation capable d'évoquer ce rapport dual à l'espace. Les participants étaient ainsi amenés à expliciter les moyens d'investigation que tout lecteur active naturellement au moment de trouver sa place dans la BNU. Ils étaient aussi conduits à s'interroger sur les stratégies mises en œuvre pour maintenir, une fois installés, un « système de protection d'isolement ", un système où, précise Moles, « la séparation est cherchée et accomplie par des modes intellectuels mieux que par des portes, des cloisons ou des rideaux ${ }^{22}$. Nous ajouterons que celui-ci passe également par le positionnement du corps (par exemple, se recroqueviller sur sa table de travail, se mettre légèrement de biais, se cacher derrière l'écran de son ordinateur...), tout comme par le réglage permanent des distances interindividuelles. Il est aussi dépendant des échanges ou non de regards, le but étant surtout de voir sans être vu. C'est ce qui est apparu de manière flagrante dans les propositions des participants.

Cette visite n'a pas la valeur d'une enquête psychosociologique conduite dans les règles de l'art. Elle se veut exploratoire et ne permet que d'amorcer des réflexions sur la relation qu'entretiennent les usagers avec la BNU. En revanche, elle nous permet d'attirer l'attention sur les dimensions corporelle et sensorielle de cette relation, des dimensions qui permettent de comprendre dans toute sa subtilité la nature du confort recherchée par les usagers.

\footnotetext{
NOTES

1- Un travail développé avec Dominique Laburte, enseignantarchitecte à l'ENSAS. Cf. Lebois, Valérie et Laburte, Dominique, " L'engagement corporel, une démarche du projet d'architecture ", in Ambiances demain, actes du congrès international des ambiances, 2016, p. 455-464 [en ligne].

2- Retour sur la psychologie de l'espace d'Abraham Moles et Elisabeth Rohmer, séminaire de recherche proposé par Valérie Lebois et Frédéric Luckel, 3 mai 2017, ENSAS.

3- Cf. Lebois, Valérie et Luckel, Frédéric, « Abraham Moles (19201992). Sa place dans l'entrée des sciences sociales à l'école d'architecture de Strasbourg " avec Frédéric Luckel, in Carnet de recherches du Comité d'histoire du ministère de la Culture sur les politiques, les institutions et les pratiques culturelles, 24 avril 2017 [en ligne].
} 


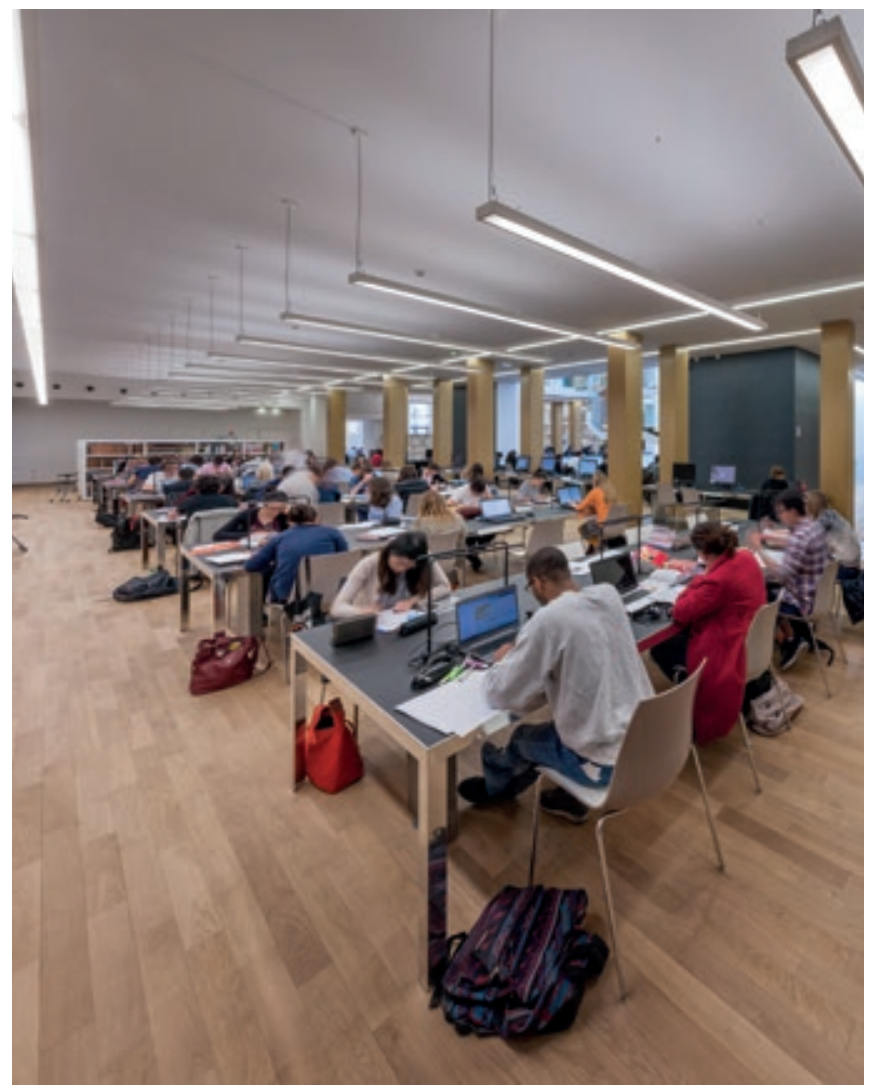

Postures et positionnements

4- Citons les principaux : Moles, Abraham et Rohmer, Elisabeth, Psychologie de l'espace, Paris, Casterman, 1972 ; idem, Labyrinthes du vécu, Paris, Librairie des Méridiens, 1982 ; idem, Psychosociologie de l'espace. Textes rassemblés, mis en forme et présentés par Victor Schwach, Paris, L'Harmattan, 1998.

5- Moles, Abraham et Rohmer, Elisabeth, Psychologie de l'espace, Casterman, 1973, p. 39

6- Moles, Abraham et Rohmer, Elisabeth, op. cit., p. 105

7- Ibid., p. 118

8- Ibid., p. 121

9- Ibid., p. 116

10- Ibid., p. 3

$11-$ Ibid., p. 122

12- Moles, Abraham et Rohmer, Elisabeth, Labyrinthes $d u$ vécu, op. cit., p. 7

13- Franqueville, Pierre, "Vers une bibliothèque d'univers ", in Revue de l'Association des bibliothécaires de France, $\mathrm{n}^{\circ}$ 47/48, décembre 2009, p. 14

14- Servet, Mathilde, « Les bibliothèques troisième lieu », in Bulletin des bibliothèques de France, t. 55, no 4, 2010, p. 57-66

15- Cf. Anne Bossé, La visite, une expérience spatiale, Presses universitaires de Rennes, 2015

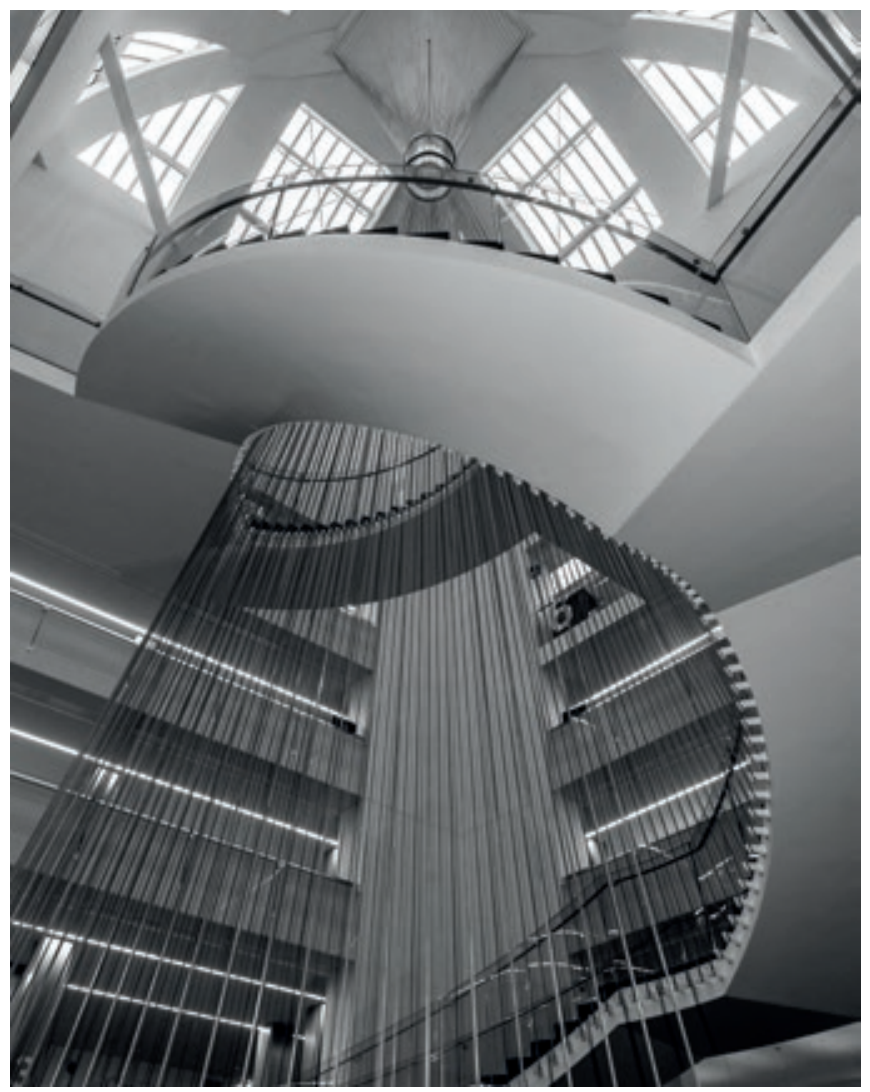

La bibliothèque comme labyrinthe

16- Moles, Abraham et Rohmer, Elisabeth, Psychosociologie de l'espace, op. cit., p. 124

17- Scoffier, Richard, Bibliothèques, Université populaire du Pavillon de l'Arsenal, samedi 18 février 2017 [en ligne]

18- Pour rappel, l'objectif de la structure panoptique est de permettre à un gardien, logé dans une tour centrale, d'observer tous les prisonniers, enfermés dans des cellules individuelles autour de la tour, sans que ceux-ci puissent savoir s'ils sont observés.

19- Scoffier, Richard, Bibliothèques, op. cit.

20- Cf. le dossier « Perception et expérience vivante des formes ", in Cahiers d'IFMA-France, $\mathrm{n}^{\circ}$ 10, juin 2009 ; Bonnaud, Xavier, « Les univers sensoriels de l'architecture contemporaine ", in Bonnaud, Xavier et Younès, Chris, Architecture et perception, La Découverte, 2012.

21- Moles, Abraham et Rohmer, Elisabeth, « Autobiographie d'Abraham Moles. Le cursus scientifique d'Abraham Moles ", in Bulletin de micropsychologie, $\mathrm{n}^{\text {os }} 28$ et 29, mars et juillet 1996, p. 28

22- Moles, Abraham et Rohmer, Elisabeth, Psychologie de l'espace, op. cit., p. 95 